\title{
A Case of Amyotrophic Lateral Sclerosis Presented as Oropharyngeal Dysphagia
}

\author{
Eun Ji Noh, MD, Moo In Park, MD*, Seun Ja Park, MD, Won Moon, MD and Hyun Joo Jung, MD \\ Department of Internal Medicine, Kosin University College of Medicine, Busan, Korea
}

Amyotrophic lateral sclerosis is a rare disease. It is a fatal neurodegenerative disease characterized by progressive muscular paralysis reflecting degeneration of motor neurons which leads to muscle weakness and muscle wasting. Respiratory failure limits survival to 2-5 years after disease onset. Several clinical manifestations including dysphagia can result in reductions in both the quality of life and life expectancy. Dysphagia occurs at onset in about one third of case, although generally it occurs in later stage of the disease. Evaluation of dysphagia includes video-fluoroscopic swallow study, radiological esophagogram, flexible endoscopic examination, ultrasound examination, conventional manometry and electromyography. We report a case of amyotrophic lateral sclerosis in a 54-year-old man presenting oropharyngeal dysphagia which was diagnosed by high resolution esophageal manometry presenting abnormality of the upper esophageal sphincter.

(J Neurogastroenterol Motil 2010;16:319-322)

Key Words

Oropharyngeal dysphagia, Amyotrophic lateral sclerosis, High resolution manometry

\section{Introduction}

Amyotrophic lateral sclerosis (ALS) or Lou gehrig's disease is a relatively rare disease. The incidence is about 1-2 per $100,000 .{ }^{1}$ It is a fatal neurodegenerative disease characterized by progressive muscular paralysis reflecting degeneration of motor neurons in the motor cortex, corticospinal tract, brainstem and spinal cord. ${ }^{2,3}$ It leads to muscle weakness and muscle wasting due to unsend messages to muscles. Respiratory failure limits survival to 2-5 years after disease onset. ${ }^{4}$ Dysphagia is one of the frequent features of ALS and can result in reductions in life expectancy and quality of life. It can lead to nutritional deficiency and aspiration pneumonia. This symptom occurs at onset of dis- ease in about one third of case, although generally it occurs in later stage of the disease. ${ }^{5,6}$

Neurogenic dysphagia can be evaluated by video-fluoroscopic swallow study, radiological esophagogram, flexible endoscopic examination, ultrasound examination, conventional manometry and electromyography. ${ }^{7}$ High resolution manometry (HRM) has been recently developed and defined the intraluminal pressure of the esophagus more completely than conventional manometry and is very useful and safe in evaluating oropharyngeal, neurogenic dysphagia. ${ }^{8-12}$

We report a case of an amyotrophic lateral sclerosis in a 54-year-old man presenting oropharyngeal dysphagia which was evaluated by high resolution esophageal manometry.

Received: June 16, 2010 Revised: July 14, 2010 Accepted: July 16, 2010

(c) This is an Open Access article distributed under the terms of the Creative Commons Attribution Non-Commercial License (http://creativecommons. org/licenses/by-nc/3.0) which permits unrestricted non-commercial use, distribution, and reproduction in any medium, provided the original work is properly cited.

*Correspondence: Moo In Park, MD

Department of Internal Medicine, Kosin University College of Medicine, 34 Amnam-dong, Seo-gu, Busan 602-702, Korea

Tel: +82-51-990-6719, Fax: +82-51-990-5055, E-mail: mipark@ns.kosinmed.or.kr

Financial support: This study was supported in part by a fund from the Institute of Kosin Medical Science.

Conflicts of interest: None. 


\section{Case Report}

A 54-year-old man was admitted to the hospital complaining dysphagia and hoarseness of 3 months. He developed weakness in both arms 1 year before the admission, which has disabled him for lifting a heavy load over his head. He experienced shortness of breath when going up a hill or stairs. But he had no difficulty in swallowing during the initial hospital visit. Gradually, he developed difficulty in lifting his hands over his head and repeatedly stopped level walking due to shortness of breath. Thereafter, from 3 months before admission, he could not lift up his arms to the chest level, and dysphagia occurred. He had difficulty in swallowing porridge and water, and had to spit because he could not swallow his saliva. He couldn't sleep well because of shortness of breath, which led him to stay awake every night for 1 to 2 hours. He was admitted for evaluating dysphagia and hoarseness. There was no specific findings on medical history or familial history. Vital signs were stable. He was alert, but appeared chronically ill. Nutritional status was poor and he complained of swallowing difficulty. Both upper proximal extremities showed Medical Research Council grade 3-4 weakness and especially, both arm abduction were graded 1-2.

Laboratory studies showed the following parameters: white blood cell count $10,900 / \mathrm{mm}^{3}$, hemoglobin $16.1 \mathrm{~g} / \mathrm{L}$, platelets $434,000 / \mathrm{mm}^{3}$, serum sodium $133 \mathrm{mEq} / \mathrm{L}$, serum potassium 4.2 $\mathrm{mEq} / \mathrm{L}$, serum creatinine $1.0 \mathrm{mg} / \mathrm{dL}$, serum blood urea nitrogen $12 \mathrm{mg} / \mathrm{dL}$, serum albumin $4.3 \mathrm{~g} / \mathrm{dL}$, serum lactate dehydrogenase $307 \mathrm{IU} / \mathrm{L}$, serum erythrocyte sedimentation rate $20 \mathrm{~mm} / \mathrm{hr}$ and serum C-reactive protein $3.9 \mathrm{mg} / \mathrm{dL}$. In addition, rheumatoid, anti-nuclear antibody, anti-DNA antibody, anti-neutrophil cytoplasmic antibody, anti-extractable nuclear antibody, anti-Ro/SSA antibody and anti-La/SSB antibody showed negative results with no abnormality on the chest and abdominal CT scan.

Neurologic examination revealed jaw jerk signs and positive Hoffman sign. The deep tendon reflexes of both the upper and lower limbs were positive for upper motor signs with atrophy of the tongue. Patient presented symptoms such as swallowing difficulties for liquids and solids, bulbar weakness signs of hoarseness, muscle atrophy and weakness in the trunk, neck, back and both proximal upper limbs for lower motor signs. Brain MRI was recommended but refused by the patient.

Esophagogastroduodenoscopy revealed neither a mucosal abnormality nor an unstained area by lugol spray on the esophagus and the scope passed easily through the lumen. However, we had difficulty in performing endoscopy because of the hypoxemia developed during the procedure and poor cooperation of the patient. Esophagogram showed the retention of contrast agent in the upper esophageal sphincter, which did not pass down after swallowing, and thus, remained in the pharynx and was aspirated into the airway. Nerve conduction velocity revealed sensorimotor polyneuropathy, and electromyography showed increased insertional activity, fibrillation on rest and a positive sharp wave in the first dorsal interosseous muscle, extensor digitorum communius muscle, biceps brachii muscle, deltoid muscle and tibiali anterior muscle. Additionally, the interference pattern was decreased in all of these muscles. Acute degenerations and chronic changes in widespread distribution confirmed the lower motor neuron injury. On video fluoroscopic swallow study (VFSS), movements of the tongue in the oral phase was intact and there was no sign of tongue apraxia. Swallowing reflex in the pharyngeal phase was slightly impaired and aspiration without coughing was observed for thin liquid, thick liquid, semisolid and solid. Grade 2 and 3 residue was observed in the valleculae and piriform sinus, respectively (Fig. 1).

High resolution esophageal manometry were done in a supine position after at least 8 hours of fast. The manometric protocol included a 5-minute period to assess basal sphincter pressure and 10 series of $5-\mathrm{mL}$ water swallows. The patient performed only four successful 5-mL water swallows because of the dysphagia. On HRM, Upper esophageal sphincter (UES) was relaxed after

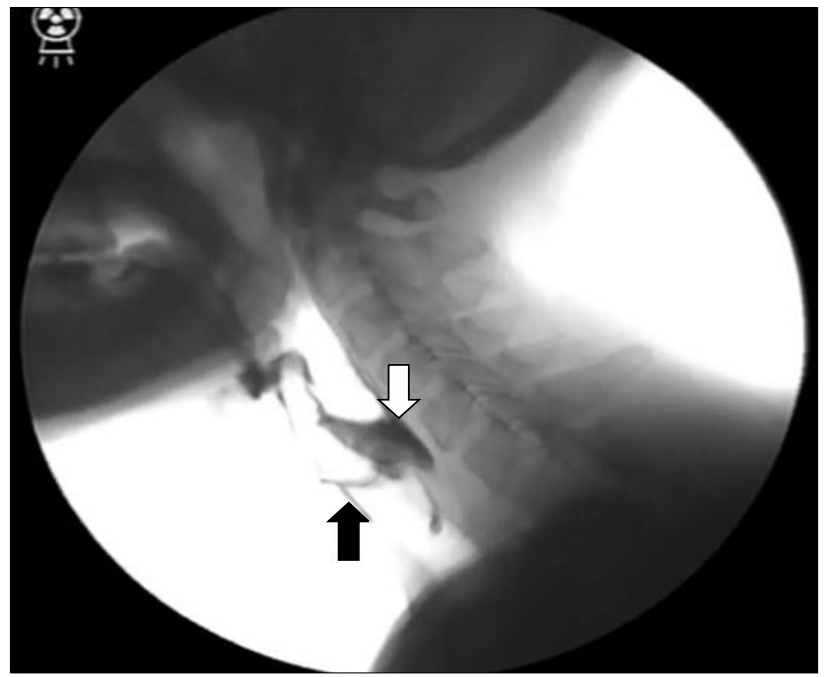

Figure 1. Video-fluoroscopic swallow study shows residues in valleculae and piriform sinus (white arrow) and tracheal aspiration (black arrow). 


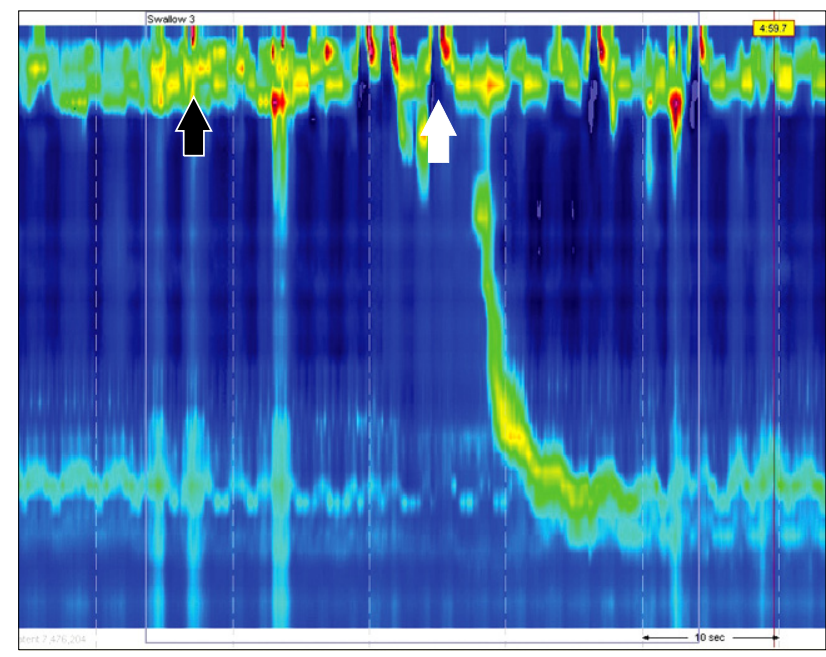

Figure 2. High resolutional esophageal manometric findings. Upper esophageal sphincter (UES) does not show relaxation on swallowing (white arrow). After trying several times, the UES becomes relaxed (black arrow). No other abnormal esophageal motility is found.

several attempted swallowing as indicated by several pharyngeal contractions before the relaxation. Manometric data were initially analyzed using ManoView analysis software (Sierra Scientific Instruments, Los angel, CA, USA). When $5 \mathrm{~mL}$ of water was administered orally, it passed to the esophagus after several trials, and the pharyngeal contraction wave showed a weak movement with low amplitude. Relaxation of the lower esophageal sphincter was sufficient (Fig. 2).

This case was diagnosed clinically as "definite ALS" based on the diagnostic criteria of the revised 2000 'Airlie House' criteria, the World Federation of Neurology (WFN), in which positive upper motor neuron and lower motor signs in the 3 lesions (bulbar, cervical and thoracic) were confirmed. ${ }^{2}$ Abnormality in the lower motor neuron presented as diffuse denervation across the motor neuron axis on the electromyography. These findings supported diagnosing the patient with ALS and swallowing difficulty was confirmed through the VFSS and HRM.

At the present time, supportive care to relieve symptoms and improve quality of life, with bilevel positive airway pressure to assist in weakened breathing during sleep were applied to the patient and percutaneous endoscopic gastrostomy was performed to avoid airway aspiration due to swallowing difficulty.

\section{Discussion}

Dysphagia is one of the most important prognostic factors in ALS. This symptom occurs at onset of the disease in about one third of cases, although it occurs mostly in later stage. Dysphagia causes aspiration pneumonia, nutritional deficiency and dehydration. Therefore, proper and careful evaluation and follow-up for swallowing function is essential. ${ }^{5}$ Pathophysiological mechanism of dysphagia in ALS is as follows. First, submental muscle activity of the laryngeal elevators is significantly prolonged. Second, opening of the cricopharyngeal sphincter muscle is delayed or the muscle closes prematurely. Third, there is a significant lack of coordination between the laryngeal elevator muscles and the cricopharyngeal sphincter muscle. ${ }^{13}$ Altogether, these abnormalities may lead to decreased ability to initiate complete reflex swallowing in ALS as demonstrated in the HRM study in this patient.

An objective method for the evaluation of dysphagia in ALS is variable, but should be uncomplicate, repeatedly reproducible and easy to perform. VFSS is an objective, easily repeatable, non-invasive test and can provide direct assessments of oropharyngeal stage of swallowing. But, it also has the disadvantage of possible risk for aspiration of contrast containing food, exposure to radiation and inability to measure the weakness of muscles related to swallowing quantitatively. ${ }^{6}$ Although conventional esophageal manometry has superiority in the evaluation of functional esophageal disease, its sensor to the pharynx and upper esophagus is limited and difficult to interpret. And it is limited with not enough response time to evaluate the pharyngeal and UES with incorrect measurements due to movement artifacts in the pharynx during swallowing. High resolutional anometry is ideally suited for study of the pharynx and UES by the availability of a catheter with a large number of closely spaced, circumferentially integrated and non-perfused transducers overcoming the chief limitations of conventional manometry. ${ }^{10-12}$ It can be used as a useful diagnostic method combined with VFSS for evaluating oropharyngeal dysphagia in ALS.

Positional changes during meal, dietary modifications and augmented feeding technique were provided to treat dysphagia of the patient. Except for augmented feeding technique, they are auxiliary methods. Percutaneous endoscopic gastrostomy was performed due to insufficient efficacy of the auxiliary method. ${ }^{14}$

We report a patient with ALS who underwent HRM due to oropharyngeal dysphagia, which is a safe and good diagnostic method for the evaluations of the function of the pharyngeal mus- 
cles and UES orophayngeal dysphagia.

\section{References}

1. Eisen A. Amyotrophic lateral sclerosis: a 40-year personal perspective. J Clin Neurosci 2009;16:505-512.

2. Wijesekera P, Nigel PN. Amyotrophic lateral sclerosis. Orphanet J Rare Dis 2009;4:3.

3. Rowland LP, Shneider NA. Amyotrophic lateral sclerosis. N Engl J Med 2001;344:1688-1700.

4. Van Damme P, Robberecht W. Recent advances in motor neuron diseases. Curr Opin Neurol 2009;22:486-492.

5. Kühnlein P, Gdynia HJ, Sperfeld AD, et al. Diagnosis and treatment of bulbar symptoms in amyotrophic lateral sclerosis. Nat Clin Pract Neurol 2008;4:366-374.

6. Gonzalez-Fernandez M, Daniels SK. Dysphagia in stroke and neurologic disease. Phys Med Rehabil Clin N Am 2008;19:867-888.

7. Olszewski J. Causes, diagnosis and treatment of neurogenic dysphagia as an interdisciplinary clinical problem. Otolaryngol Pol 2006;60: 491-500.

8. Ghosh SK, Pandolfino JE, Zhang Q, Jarosz A, Kahrilas PJ.
Deglutitive upper esophageal sphincter relaxation: a study of 75 volunteer subjects using solid-state high-resolution manometry. Am J Physiol Gastrointest Liver Physiol 2006;291:G525-G531.

9. Pandolfino JE, Ghosh SK, Rice J, Clarke JO, Kwiatek MA, Kahrilas PJ. Classifying esophageal motility by pressure topography characteristics: a study of 400 patients and 75 controls. Am J Gastroenterol 2008;103:27-37.

10. Ayazi S, Crookes PF. High-resolutional esophageal manometry: using technical advances for clinical advantage. J Gastrointest Surg 2010;14:S24-S32.

11. Kahrilas PL, Ghosh SK, Pandolfino JE. Esophageal motility disorders in terms of pressure topography. J Clin Gastrenterol 2008; 42:627-635.

12. Park MI. Clinical usefulness of high-resolution manometry. Korean J Neurogastroenterol Motil 2009;15:107-115.

13. Ertekin C, Aydogdu I, Yuceyar N, Kiylioglu N, Tarlaci S, Uludag B. Pathophysiological mechanisms of oropharyngeal dysphagia in amyotrophic lateral sclerosis. Brain 2000;123:125-140.

14. Kuhnlein P, Gdynia HJ, Sperfeld AD, et al. Diagnosis and treatment of bulbar symptoms in amyotrophic lateral sclerosis. Nat Clin Pract Neurol 2008;4:366-374. 